\title{
Polyaromatic hydrocarbon oxyradical stability
}

\author{
Dmitry Yu. Zubarev ${ }^{a}$, Neil Robertson ${ }^{b}$, Dominik Domin ${ }^{a, c}$,
} Jarrod McClean $^{a}$, Jin-Hua Wang ${ }^{a, d}$, William A. Lester, Jr. ${ }^{a, c, *}$, Russell Whitesides $^{b, e}$, Xiao-Qing You ${ }^{b}$, Michael Frenklach $^{b, e}$

a Kenneth S. Pitzer Center for Theoretical Chemistry, Department of Chemistry,

University of California at Berkeley, Berkeley, California 94720-1460, USA

${ }^{b}$ Department of Mechanical Engineering, University of California at Berkeley, Berkeley, California 94720-1740, USA

${ }^{c}$ Chemical Sciences Division, Lawrence Berkeley National Laboratory, Berkeley, California 94720, USA

dState Key Laboratory of Superhard Materials, Jilin University, Changchun 130012, China

${ }^{e}$ Environmental Energy Technologies Division, Lawrence Berkeley National Laboratory, Berkeley, California 94720

Received 13 January 2010; Accepted (in revised version) 20 January 2010; Available online 15 February 2010

\begin{abstract}
A simple connection is established between aromaticity and overall stability of five-ring linear polyaromatic hydrocarbon oxyradicals. A chemically intuitive model is developed that exhibits a linear trend between an inexpensive geometric measure of aromaticity and the energetic stability of a model graphene edge with oxygen at different positions. It is believed to be an important step in establishing reaction pathways of soot and graphene oxidation.
\end{abstract}

PACS: $31.15 . \mathrm{A}-$, 73.22.Pr

Key words: graphene edge, polyaromatic hydrocarbons, oxidation, aromaticity

\section{Introduction}

While development of alternative sources of energy and reduction of carbon emissions remain crucial in the battle for environmental and public health, the fact remains that combustion reactions and carbon fuels will be prevalent for some time. As such, it is critical to investigate means of mitigating the dangers inherent to some of these methods.

\footnotetext{
*Corresponding author. Email address: walester@lbl.gov (W. A. Lester, Jr.)
} 
One of the most harmful byproducts of these processes is soot, which is known to consist of polyaromatic hydrocarbon fragments. Additionally, one of the primary pathways by which soot production is alleviated is oxidation. Past theoretical studies of soot oxidation have focused on the model cases of oxidation reactions of one-ring aromatics [1-4] and oxygen chemisorption at selective sites of two- and three-ring aromatics [5-8].A crucial first step towards the understanding of these pathways is knowledge of the stability of oxyradical intermediates.

Many of the systems of interest are, unfortunately, too large to treat by highly accurate methods. It is the goal of this work to develop an efficient and accurate model based on intuitive notions of aromaticity for determining the stability of polycyclic aromatic hydrocarbon oxyradical fragments.

\section{Computational details}

The geometries of oxyradical derivatives of pentacene depicted in Fig. 1 were optimized at the UB3LYP/6-311G(d, p) level of theory [9-12] in the Gaussian 03 software package [13]. The results of these calculations were verified by ab initio perturbation theory (RO-RI-SOS-MP2/6-311G(d, p)) at the UB3LYP geometries in the Q-Chem [14] software package. The expectation value of the $S^{2}$ operator was found to be 0.75 for all oxyradicals as a consequence of the restricted open shell formalism. A normal mode calculation confirmed all structures represented an energy minimum. A factor [15] of 0.967 was used to scale vibrational frequencies and zero-point energies (ZPE).
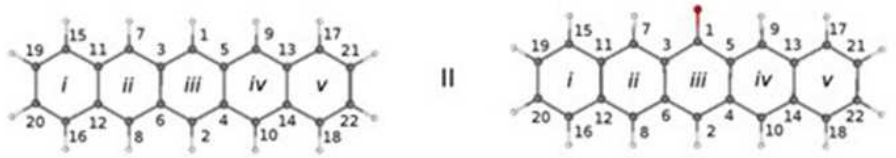

III

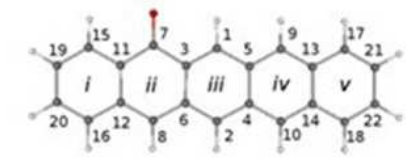

IV
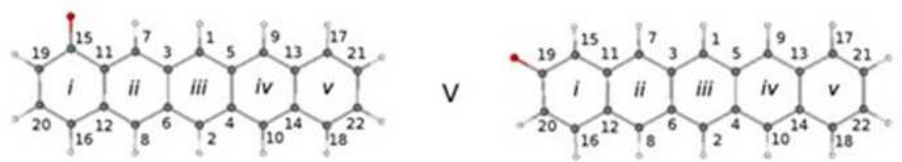

Figure 1: Structures of the pentacene molecule (I) and pentacene oxyradicals with the oxygen atom in different positions (II - V). Connections between atoms are drawn on the basis of interatomic distance. Upper case Roman numerals designate oxyradicals with different $\mathrm{O}$ atom positions; lower case italic Roman numerals designate six-atom rings; Arabic numerals enumerate $C$ atoms.

A simple geometric measure of aromaticity known as the harmonic oscillator model of aromaticity (HOMA) [16], was used. HOMA offers an inexpensive evaluation of local aromatic character based on a simple comparison to an ideal aromatic benzene. HOMA 
Table 1: Enthalpies of formation and entropies of pentacene oxyradicals at $298.15 \mathrm{~K}$ relative to those of pentacene oxyradical II at B3LYP/6-311G $(d, p)$ level of theory; relative energies of pentacene oxyradicals at 0 $\mathrm{K}$ at B3LYP/6-311G $(d, p)$ and MP2/6-311G $(\mathrm{d}, \mathrm{p}) / / \mathrm{B} 3 \mathrm{LYP} / 6-311 \mathrm{G}(d, p)$ (in parenthesis) levels of theory.

\begin{tabular}{|c|c|c|c|c|}
\hline Pentacene oxyradical & II & III & IV & V \\
\hline Enthalpy (kcal/mol) relative to II & 0 & 2.8 & 13.6 & 16.5 \\
Entropy (cal/mol K) relative to II & 0 & 3.0 & 3.2 & 3.7 \\
$E_{\text {rel }}(\mathrm{kcal} / \mathrm{mol})$ & $0(0)$ & $2.9(3.5)$ & $14.1(14.6)$ & $17.1(17.7)$ \\
\hline
\end{tabular}

is formally defined as follows

$$
\text { HOMA }=1-\frac{a}{n} \sum_{i=1}^{N}\left(r_{c c}-r_{c c-i d e a l}\right)^{2}
$$

where $r_{c c}$ is the computed carbon-carbon bond length, $r_{c c-i d e a l}$ is the ideal carbon-carbon bond length in benzene, $\mathrm{n}$ is the number of bonds in the ring, and a was chosen such that HOMA = 1 for the aromatic form of benzene, and approaches 0 for a Kekule form of benzene. It is possible to use the sum of the HOMA indices for individual rings in the PAH (cumulative HOMA) to characterize global aromaticity of a molecule [17-19].

An additional measure of aromaticity, the nucleus independent chemical shifts (NICS) $[20,21]$ metric, was used for comparison with HOMA values. NICS offers a straightforward measure of aromaticity based on magnetic shielding in the center of a ring. It is calculated at the geometric center of the ring and $0.5 \AA$ and $1.0 \AA$ above the plane of the ring, yielding NICS(0.5) and NICS(1). Negative values of NICS are indicative of aromaticity while positive values suggest anti-aromaticity. Values close to zero are typical for most non-aromatic systems. The present work primarily utilized the out-of-plane component of the NICS tensor $\left(\mathrm{NICS}_{z z}\right)$ at $1.0 \AA$ from the plane of the ring as a measure of aromaticity because of its better performance for planar rings [22].

\section{Results and discussion}

The enthalpies of formation and entropies of the four pentacene oxyradicals at $298.15 \mathrm{~K}$ relative to oxyradical II are listed in Table 1. The enthalpy of formation of oxyradical II is the most favorable energetically, while that of oxyradical $\mathrm{V}$ is the least favorable of the four. To assess thermodynamic stability, we calculated the standard Gibbs free energy of the four pentacene oxyradicals relative to oxyradical II, with results shown in Fig. 2.

Examination of the data displayed in Fig. 2 indicates that below $1000 \mathrm{~K}$ the stability of the four oxyradicals follows the trend of the relative energies at both B3LYP and MP2 levels of theory, i.e., is in the order: II > III > IV > V, while above $1000 \mathrm{~K}$ oxyradical III becomes more stable than II. This is indicative of the entropic favorability of pentacene oxyradical III at higher temperatures. 


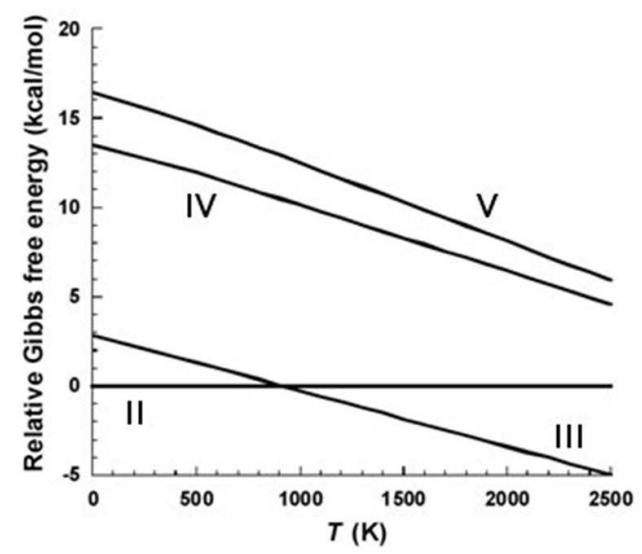

Figure 2: Standard Gibbs free energy of pentacene oxyradicals relative to that of pentacene oxyradical II as a function of temperature at B3LYP/6-311G $(d, p)$ level of theory.

The aromatic character predicted by HOMA is compared with the value predicted by NICSzz for each ring in Table 2 and found to be in good agreement. This supports HOMA as a reliable indicator of local aromaticity in the oxyradicals systems studied.

The relative energetic stabilities calculated by DFT are in agreement with the values calculated by $a b$ inito perturbation theory (RO-RI-SOS-MP2/6-311G $(d, p)$ ) at the UB3LYP geometries. The ZPE-corrected relative energies of oxyradicals II-V (at the UB3LYP/6$311 \mathrm{G}(d, p)$ level of theory) are plotted against cumulative HOMA in Fig. 3. A linear trend between the two is observed, suggesting a direct correlation between energetic stability and degree of aromatic character.

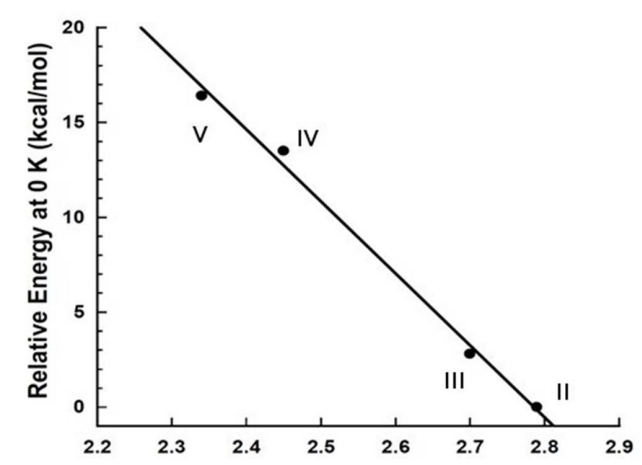

Figure 3: The relative energies of oxyradicals at $0 \mathrm{~K}$ plotted against the corresponding cumulative HOMA value. 
Table 2: Assessment of local aromaticity: values of HOMA and NICS ${ }^{z z}$ indices for structures I - V and prototypical aromatic benzene and naphthalene molecules.

\begin{tabular}{|c|c|c|c|c|c|c|c|c|c|c|}
\hline \multirow[t]{2}{*}{ Structure } & \multicolumn{2}{|c|}{ Ring $i$} & \multicolumn{2}{|c|}{ Ring $i i$} & \multicolumn{2}{|c|}{ Ring iii } & \multicolumn{2}{|c|}{ Ring $i v$} & \multicolumn{2}{|c|}{ Ring $v$} \\
\hline & HOMA & $\mathrm{NICS}_{z z}^{a}$ & HOMA & $\operatorname{NICS}_{z z}^{a}$ & HOMA & $\operatorname{NICS}_{z z}^{a}$ & HOMA & $\operatorname{NICS}_{z z}^{a}$ & HOMA & $\mathrm{NICS}_{z z}^{a}$ \\
\hline I & 0.49 & -4 & 0.57 & -17 & 0.59 & -22 & 0.57 & -17 & 0.49 & -4 \\
\hline & & -13 & & -27 & & -31 & & -27 & & -13 \\
\hline & & -21 & & -33 & & -37 & & -33 & & -21 \\
\hline II & 0.77 & -7 & 0.71 & -1 & -0.17 & 25 & 0.71 & -1 & 0.77 & -7 \\
\hline & & -16 & & -11 & & 17 & & -11 & & -16 \\
\hline & & -24 & & -20 & & 4 & & -20 & & -24 \\
\hline III & 0.89 & -3 & -0.12 & 24 & 0.57 & 2 & 0.69 & -10 & 0.67 & -7 \\
\hline & & -12 & & 16 & & -7 & & -19 & & -16 \\
\hline & & -20 & & 3 & & -17 & & -27 & & -24 \\
\hline V & 0.03 & 16 & 0.43 & 10 & 0.58 & -3 & 0.67 & -11 & 0.63 & -7 \\
\hline & & 8 & & 1 & & -12 & & -21 & & -15 \\
\hline & & -3 & & -10 & & -21 & & -28 & & -23 \\
\hline Benzene & & 0.99 & -14 & na & na & na & na & na & na & na \\
\hline $\begin{array}{l}\text { Naphth- } \\
\text { alene }\end{array}$ & & 0.79 & -13 & 0.79 & -13 & na & na & na & na & na \\
\hline
\end{tabular}

${ }^{a}$ Three NICS $_{z z}$ values are calculated at $0.0 \AA, 0.5 \AA$, and $1.0 \AA$ above the ring, yielding NICS (0.0), NICS (0.5) and NICS (1), respectively.

\section{Conclusions}

The relative stability of the polyaromatic oxyradical species studied exhibits a strong linear correlation between the relative energetic stabilities and the aromatic character predicted by HOMA. This offers an inexpensive and chemically intuitive model to explain the stability trends observed. The simple geometric nature of the model suggests a natural extension to larger systems which are intractable by high accuracy quantum mechanical calculations or a prediction by an exponentially growing number of resonance structures. An in-depth analysis of the present work and extension to fundamentals in local electronic structure is available [23].

Acknowledgments. RW, DD, WAL, and MF were supported by the Director, Office of Energy Research, Office of Basic Energy Sciences, Chemical Sciences, Geosciences and Biosciences Division of the US Department of Energy, under Contract No. DEAC03-76F00098. NR, XY, and MF were supported by the US Army Corps of Engineers, Humphreys Engineering Center Support Activity, under Contract No. W912HQ-07-C0044. DYZ was supported by the National Science Foundation under grant NSF CHE0809969. JW is a UC Berkeley visitor supported by a stipend from the China Scholarship 
Council. JM was supported in part by the Dreyfus foundation.

\section{References}

[1] K. Brezinsky, Prog. Energy Combust. Sci. 12 (1986) 1.

[2] S. Olivella, A. Sole, and A. Garcia-Raso, J. Phys. Chem. 99 (1995) 10549.

[3] M. Fadden and C. M. Hadad, J. Phys. Chem. A 104 (2000) 3004.

[4] Z. F. Xu and M. C. Lin, J. Phys. Chem. A 110 (2006) 1672.

[5] A. Montoya, F. Mondragon, and T. N. Truong, Fuel Proc. Technol. 77-78 (2002) 125.

[6] K. Sendt and B. S. Haynes, Proc. Combust. Inst. 30 (2005) 2141.

[7] K. Sendt and B. S. Haynes, Combust. Flame 143 (2005) 629.

[8] K. Sendt and B. S. Haynes, J. Phys. Chem. A 109 (2005) 3438.

[9] C. T. Lee, W. T. Yang, and R. G. Parr, Phys. Rev. B 37 (1988) 785.

[10] A. D. Becke, J. Chem. Phys. 98 (1993) 5648.

[11] R. Ditchfie, W. J. Hehre, and J. A. Pople, J. Chem. Phys. 54 (1971) 724.

[12] W. J. Hehre, R. Ditchfie, and J. A. Pople, J. Chem. Phys. 56 (1972) 2257.

[13] M. J. Frisch, G. W. Trucks, H. B. Schlegel, et al., Gaussian 03, Rev. A.1 ed. (Gaussian, Inc., Pittsburgh PA, 2003).

[14] Y. Shao, L. F. Molnar, Y. Jung, et al., Phys. Chem. Chem. Phys. 8 (2006) 3172.

[15] R. D. Johnson, III. National Institute of Standards and Technology, Release 14, Sept. 2006 (NIST publishing, Rockville, USA, 2006).

[16] J. Kruszewski and T. M. Krygowski, Tetrahedron Lett. 13 (1972) 3839.

[17] P. V. Schleyer and H. J. Jiao, Pure and Appl. Chem. 68 (1996) 209.

[18] J. Aihara and H. Kanno, J. Phys. Chem. A 109 (2005) 3717.

[19] M. K. Cyranski, T. M. Krygowski, A. R. Katritzky, and P. V. Schleyer, J. Org. Chem. 67 ( 2002) 1333.

[20] P. V. Schleyer, C. Maerker, A. Dransfeld, H. J. Jiao, and N. Hommes, J. Am. Chem. Soc. 118 (1996) 6317.

[21] Z. F. Chen, C. S. Wannere, C. Corminboeuf, R. Puchta, and P. V. Schleyer, Chem. Rev. 105 (2005) 3842.

[22] H. Fallah-Bagher-Shaidaei, C. S. Wannere, C. Corminboeuf, R. Puchta, and P. V. Schleyer, Organic Lett. 8 (2006) 863.

[23] D. Zubarev, N. Robertson, D. Domin, J. McClean, J. H. Wang, W. A. Lester, R. Whitesides, X. Q. You, and M. Frenklach, J. Phys. Chem. C, DOI: 10.1021/jp9058615 